\title{
Fibre Picard Operators on Gauge Spaces and Applications
}

\author{
I. A. Rus, A. Petruşel, and M. A. Şerban
}

\begin{abstract}
The purpose of this paper is to prove some fibre Picard operator principles in gauge spaces. As application, the differentiability with respect to parameters of the solution of a Volterra functional-integral equation is discussed.
\end{abstract}

Keywords. Fixed point, fibre Picard operator, gauge space, integral equation, data dependence

Mathematics Subject Classification (2000). Primary 47H10, secondary 54H25, $45 \mathrm{~N} 05$

\section{Introduction}

Throughout this paper we shall follow the standard terminologies and notations in Nonlinear Analysis. For the convenience of the reader we shall recall some of them.

Let $X$ be a nonempty set and $T: X \rightarrow X$ an operator. Then $T^{0}:=1_{X}$, $T^{1}:=T, T^{n+1}:=T \circ T^{n}, n \in N$, denote the iterate operators of the operator $T$. Also, by $F_{T}:=\{x \in X \mid T(x)=x\}$ we will denote the fixed point set of the operator $T$.

By $(X, \rightarrow)$ we will denote an L-space. For example, Hausdorff topological spaces, metric spaces, generalized metric spaces (in Perov' sense: $d(x, y) \in \mathbb{R}_{+}^{m}$, in Luxemburg-Jung' sense: $d(x, y) \in \mathbb{R}_{+} \cup\{+\infty\}, d(x, y) \in K, K$ a cone in an ordered Banach space, $d(x, y) \in E, E$ an ordered linear space with a notion of linear convergence, etc.), 2-metric spaces, D-R-spaces, probabilistic metric spaces, gauge spaces, syntopogenous spaces, have a natural structure of L-spaces ( $[2,4,6,9,11,14,15,21,27,28,30])$. For more details see M. Fréchet [16], L. M. Blumenthal [10] and I. A. Rus [26].

In this paper, we need the following notions (I. A. Rus [26]):

I. A. Rus, A. Petruşel, and M. A. Şerban: Department of Applied Mathematics, Babeş-Bolyai University, 400084 Cluj-Napoca, Romania;

\{iarus, petrusel,mserban\}@math.ubbcluj.ro 
Definition 1.1. Let $(X, \rightarrow)$ be an L-space. An operator $T: X \rightarrow X$ is a Picard operator (briefly PO) if:

(i) $F_{T}=\left\{x^{*}\right\}$;

(ii) $T^{n}(x) \rightarrow x^{*}$ as $n \rightarrow \infty$, for all $x \in X$.

Definition 1.2. Let $(X, \rightarrow)$ be an L-space. An operator $T: X \rightarrow X$ is a weakly Picard operator (briefly WPO) if the sequence $\left(T^{n}(x)\right)_{n \in N}$ converges for all $x \in X$ and the limit (which may depend on $x$ ) is a fixed point of $T$.

If $T: X \rightarrow X$ is a WPO, then we may define the operator $T^{\infty}: X \rightarrow X$ by $T^{\infty}(x):=\lim _{n \rightarrow \infty} T^{n}(x)$. Obviously $T^{\infty}(X)=F_{T}$. Moreover, if $T$ is a PO and we denote by $x^{*}$ its unique fixed point, then $T^{\infty}(x)=x^{*}$, for each $x \in X$.

The following open problem was posed by I. A. Rus (see [26, Problem 10.5]):

Fibre Picard operator problem. Let $(X, \stackrel{1}{\rightarrow})$ and $(Y, \stackrel{2}{\rightarrow})$ be two L-spaces. Let $B: X \rightarrow X$ be a PO and $C: X \times Y \rightarrow Y$ be such that $C(x, \cdot): Y \rightarrow Y$ is a PO, for every $x \in X$. Consider the triangular operator A defined as follows:

$$
A: X \times Y \rightarrow X \times Y, \quad A(x, y):=(B(x), C(x, y))
$$

In which conditions $A$ is a $\mathrm{PO}$ ?

The purpose of this paper is to give some answers to this problem in gauge spaces. As application, the differentiability with respect to parameters of the solution of a Volterra functional-integral equation is discussed.

\section{Fibre Picard operator problem}

To our knowledge, the first contribution in this respect belongs to M. W. Hirsch, C. C. Pugh in [19] (see also M. W. Hirsch, C. C. Pugh, M. Shub [20] and I. C. de Oliveira [23]).

Theorem 2.1 (Hirsch-Pugh [19]). Let $(X, d),(Y, \rho)$ be two metric spaces. Let $B: X \rightarrow X$ be an operator having an attractive fixed point $p \in X$. Let $C$ : $X \times Y \rightarrow Y$ be such that:

(a) there exists $\alpha \in] 0,1[$ such that $C(x, \cdot): Y \rightarrow Y$ is an $\alpha$-contraction, for each $x \in X$;

(b) the operator $A: X \times Y \rightarrow X \times Y, A(x, y):=(B(x), C(x, y))$ is continuous;

(c) $(Y, \rho)$ is complete.

Let $q \in Y$ be a fixed point for $C(p, \cdot)$. Then $(p, q)$ is an attractive fixed point for $A$. 
Some generalizations of Hirsch-Pugh result, as well as, other partial answers to the above open question are given in J. K. Hale, L. A. C. Ladeira [18], I. A. Rus [24,25], M. A. Şerban [30], G. Dezsö [13], C. Bacoţiu [6] and Sz. Andras [1,2].

For example, we have:

Theorem 2.2 (I. A. Rus [24]). Let $(X, d),(Y, \rho)$ be two metric spaces. Let $B: X \rightarrow X$ and $C: X \times Y \rightarrow Y$ be two operators. Consider $A: X \times Y \rightarrow X \times Y$, defined by $A(x, y):=(B(x), C(x, y))$. Suppose that:

(a) $(Y, \rho)$ is complete;

(b) $B$ is a WPO;

(c) there exists $\alpha \in] 0,1[$ such that $C(x, \cdot): Y \rightarrow Y$ is an $\alpha$-contraction, for each $x \in X$;

(d) if $\left(x^{*}, y^{*}\right) \in F_{A}$, then the operator $\left.C\left(\cdot, y^{*}\right)\right)$ is continuous in $x^{*}$.

Then $A$ is a WPO. Moreover, if $B$ is a PO, then $A$ is a PO too.

Throughout this paper, a gauge space is a set endowed with a gauge structure induced by a family $\left\{d_{i}: i \in I\right\}$ of pseudo-metrics, where $I$ is a directed set. The basic definitions and properties of gauge spaces may be found, for example, in [14].

For our first main theorem we need some auxiliary results.

Lemma 2.3. Let $\left(X,\left(d_{i}\right)_{i \in I}\right)$ be a sequentially complete Hausdorff gauge space and let $T, T_{n}: X \rightarrow X$ be operators such that:

(i) the sequence $\left(T_{n}\right)_{n \in \mathbb{N}}$ pointwise converges to $T$;

(ii) for every $i \in I$ there exists $\left.\alpha_{i} \in\right] 0 ; 1[$ such that

$$
\begin{aligned}
d_{i}\left(T_{n}(x), T_{n}(y)\right) & \leq \alpha_{i} \cdot d_{i}(x, y) \\
d_{i}(T(x), T(y)) & \leq \alpha_{i} \cdot d_{i}(x, y),
\end{aligned}
$$

for each $x, y \in X$.

Then $T, T_{n}, n \in \mathbb{N}$, are POs and the sequence $\left(T_{n} \circ T_{n-1} \circ \cdots \circ T_{0}\right)_{n \in \mathbb{N}}$ pointwise converges to $T^{\infty}$.

Proof. From (ii) and a Colojoara's theorem [12] we deduce that there exists a unique $x^{\star} \in F_{T}$, so $T^{\infty}(x)=x^{\star}$, for all $x \in X$. Let $x \in X$. We have

$$
\begin{aligned}
& d_{i}\left(\left(T_{n} \circ T_{n-1} \circ \cdots \circ T_{0}\right)(x), x^{\star}\right) \\
& \leq d_{i}\left(\left(T_{n} \circ T_{n-1} \circ \cdots \circ T_{0}\right)(x),\left(T_{n} \circ T_{n-1} \circ \cdots \circ T_{0}\right)\left(x^{\star}\right)\right) \\
&+d_{i}\left(\left(T_{n} \circ T_{n-1} \circ \cdots \circ T_{0}\right)\left(x^{\star}\right), T_{n}\left(x^{\star}\right)\right)+d_{i}\left(T_{n}\left(x^{\star}\right), x^{\star}\right) \\
& \leq \cdots \\
& \leq \alpha_{i}^{n+1} d_{i}\left(x, x^{\star}\right)+\alpha_{i}^{n} d_{i}\left(T_{0}\left(x^{\star}\right), x^{\star}\right)+\cdots+\alpha_{i} d_{i}\left(T_{n-1}\left(x^{\star}\right), x^{\star}\right) \\
&+d_{i}\left(T_{n}\left(x^{\star}\right), x^{\star}\right) .
\end{aligned}
$$


Let $a_{n}:=d_{i}\left(T_{n}\left(x^{\star}\right), x^{\star}\right)$ and $b_{n}:=\alpha_{i}^{n}$. Since $a_{n} \rightarrow 0$, as $n \rightarrow \infty$, and $\sum_{k=0}^{\infty} b_{k}<$ $\infty$, we get $\sum_{k=0}^{n} a_{k} b_{n-k} \rightarrow 0$, as $n \rightarrow \infty$ (see [24, Lemma 2.2]). This proves that

$$
d_{i}\left(\left(T_{n} \circ T_{n-1} \circ \cdots \circ T_{0}\right)(x), x^{\star}\right) \rightarrow 0 \quad(n \rightarrow \infty),
$$

for all $i \in I$. The proof is complete.

Lemma 2.4. Let $(X, \rightarrow)$ be an L-space and $\left(Y,\left(d_{i}\right)_{i \in I}\right)$ be a sequentially complete Hausdorff gauge space. Let $x_{n}, x^{\star} \in X$ and $f: X \times Y \rightarrow Y$ be an operator such that:

(i) $x_{n} \rightarrow x^{\star}$, as $n \rightarrow \infty$;

(ii) the operator $f(\cdot, y): X \rightarrow X$ is continuous, for all $y \in Y$;

(iii) for every $i \in I$ there exists $\left.\alpha_{i} \in\right] 0 ; 1[$ such that

$$
d_{i}\left(f\left(x, y_{1}\right), f\left(x, y_{2}\right)\right) \leq \alpha_{i} \cdot d_{i}\left(y_{1}, y_{2}\right), \text { for all } x \in X \text { and } y_{1}, y_{2} \in Y
$$

(we denote by $y^{\star}$ the unique fixed point of $f\left(x^{\star}, \cdot\right)$ ).

Then the sequence $\left(y_{n}\right)_{n \in \mathbb{N}}$ defined by

$$
y_{0} \in Y, \quad y_{n+1}=f\left(x_{n}, y_{n}\right) \quad(n \in \mathbb{N})
$$

converges to $y^{\star}$, for all $y_{0} \in Y$.

Proof. We consider

$$
T_{n}, T: Y \rightarrow Y, \quad T_{n}(y)=f\left(x_{n}, y\right), \quad T(y)=f\left(x^{\star}, y\right)
$$

We successively have

$$
\begin{aligned}
y_{n+1} & =f\left(x_{n}, y_{n}\right)=T_{n}\left(y_{n}\right)=T_{n}\left(f\left(x_{n-1}, y_{n-1}\right)\right)=T_{n}\left(T_{n-1}\left(y_{n-1}\right)\right) \\
& =\cdots=\left(T_{n} \circ T_{n-1} \circ \cdots \circ T_{0}\right)\left(y_{0}\right) .
\end{aligned}
$$

From Lemma 2.3 we get that

$$
\left(T_{n} \circ T_{n-1} \circ \cdots \circ T_{0}\right)\left(y_{0}\right) \rightarrow T^{\infty}\left(y_{0}\right)=y^{\star}=f\left(x^{\star}, y^{\star}\right),
$$

which means that $y_{n} \rightarrow y^{\star}$, as $n \rightarrow+\infty$.

The first main result of this paper is the following:

Theorem 2.5. Let $(X, \rightarrow)$ be an L-space and $\left(Y,\left(d_{i}\right)_{i \in I}\right)$ be a sequentially complete Hausdorff gauge space. Let $B: X \rightarrow X$ and $C: X \times Y \rightarrow Y$ be two operators. We suppose that:

(i) $B$ is a PO (we denote by $x^{*}$ its unique fixed point); 
(ii) for every $i \in I$ there exists $\left.\alpha_{i} \in\right] 0 ; 1[$ such that

$$
d_{i}\left(C\left(x, y_{1}\right), C\left(x, y_{2}\right)\right) \leq \alpha_{i} \cdot d_{i}\left(y_{1}, y_{2}\right), \text { for all } x \in X \text { and } y_{1}, y_{2} \in Y
$$

(we denote by $y^{*}$ the unique fixed point of the operator $C\left(x^{*}, \cdot\right)$ );

(iii) the operator $C\left(\cdot, y^{*}\right)$ is continuous in $x^{*}$.

Then, the operator $A: X \times Y \rightarrow X \times Y, A(x, y):=(B(x), C(x, y))$ is a PO. Moreover, $F_{A}=\left\{\left(x^{\star}, y^{\star}\right)\right\}$.

Proof. Let $x_{0} \in X$ and $y_{0} \in Y$. We show that

$$
A^{n}\left(x_{0}, y_{0}\right) \rightarrow\left(B^{\infty}\left(x_{0}\right), y^{\star}\right)=\left(x^{\star}, y^{\star}\right)
$$

where $\left\{y^{\star}\right\}=F_{C\left(B^{\infty}\left(x_{0}\right), \cdot\right)}=F_{C\left(x^{\star}, \cdot\right)}$. It is easy to check that

$$
A^{n}\left(x_{0}, y_{0}\right)=\left(B^{n}\left(x_{0}\right), y_{n}\right)
$$

where $x_{n}=B^{n}\left(x_{0}\right), y_{n+1}=C\left(x_{n}, y_{n}\right)$. From (i) we have that $x_{n}=B^{n}\left(x_{0}\right) \rightarrow x^{\star}$. Using again Lemma 2.3 for

$$
T_{n}, T: Y \rightarrow Y, \quad T_{n}(y)=C\left(x_{n}, y\right), \quad T(y)=C\left(x^{\star}, y\right)
$$

we obtain that $y_{n} \rightarrow y^{\star}$, as $n \rightarrow \infty$.

In the second part of this section we will extend Theorem 2.5 by using a Hadžić-Stanković type condition (see [17]).

Lemma 2.6. Let $\left(X,\left(d_{i}\right)_{i \in I}\right)$ be a sequentially complete Hausdorff gauge space, $j: I \rightarrow I$ and let $T, T_{n}: X \rightarrow X$ be operators such that:

(i) the sequence $\left(T_{n}\right)_{n \in \mathbb{N}}$ pointwise converges to $T$;

(ii) for every $i \in I$ there exists $\alpha_{i} \in \mathbb{R}_{+}$such that

$$
\begin{aligned}
d_{i}\left(T_{n}(x), T_{n}(y)\right) & \leq \alpha_{i} \cdot d_{j(i)}(x, y) \\
d_{i}(T(x), T(y)) & \leq \alpha_{i} \cdot d_{j(i)}(x, y),
\end{aligned}
$$

for all $x, y \in X$;

(iii) for every $i \in I$ there exists $m_{i} \in \mathbb{N}$ such that for $m \geq m_{i}$ we have

$$
\alpha_{j^{m}(i)} \leq \alpha(i)<1
$$

(iv) for every $i \in I$ there exists $p(i)>0$ such that

$$
d_{j^{m}(i)}\left(x, T_{n}(x)\right) \leq p(i)<\infty \quad \text { and } \quad d_{j^{m}(i)}(x, T(x)) \leq p(i)<\infty,
$$

for all $x \in X, n \in \mathbb{N}$ and $m \geq 0$; 
(v) for each $i \in I$ there is $\beta(i) \in I$ such that

$$
d_{j^{m}(i)}\left(x, T_{n}(x)\right) \leq d_{\beta(i)}\left(x, T_{n}(x)\right),
$$

for all $x \in X$ and $m \geq 0$.

Then $T, T_{n}, n \in \mathbb{N}$ are POs and the sequence $\left(T_{n} \circ T_{n-1} \circ \cdots \circ T_{0}\right)_{n \in \mathbb{N}}$ pointwise converges to $T^{\infty}$.

Proof. From (ii), (iii) and (iv) we deduce that there exists a unique $x^{\star} \in F_{T}$, $T^{\infty}(x)=x^{\star}$, for all $x \in X$ and $d_{j^{m}(i)}\left(x, x^{*}\right) \leq q(i, x):=q(p(i), x)<+\infty$, for all $m \geq 0$ (see [17]). Let $x \in X$. We have

$$
\begin{aligned}
d_{i}\left(\left(T_{n} \circ\right.\right. & \left.\left.T_{n-1} \circ \cdots \circ T_{0}\right)(x), x^{\star}\right) \\
\leq & d_{i}\left(\left(T_{n} \circ T_{n-1} \circ \cdots \circ T_{0}\right)(x),\left(T_{n} \circ T_{n-1} \circ \cdots \circ T_{0}\right)\left(x^{\star}\right)\right) \\
& +d_{i}\left(\left(T_{n} \circ T_{n-1} \circ \cdots \circ T_{0}\right)\left(x^{\star}\right), T_{n}\left(x^{\star}\right)\right)+d_{i}\left(T_{n}\left(x^{\star}\right), x^{\star}\right) \\
\leq & \cdots \\
\leq & \alpha_{i} \alpha_{j(i)} \ldots \alpha_{j^{n}(i)} d_{j^{n+1}(i)}\left(x, x^{\star}\right) \\
& +\alpha_{i} \alpha_{j(i)} \ldots \alpha_{j^{n-1}(i)} d_{j^{n}(i)}\left(T_{0}\left(x^{\star}\right), x^{\star}\right) \\
& +\cdots+\alpha_{i} d_{j(i)}\left(T_{n-1}\left(x^{\star}\right), x^{\star}\right)+d_{i}\left(T_{n}\left(x^{\star}\right), x^{\star}\right) .
\end{aligned}
$$

For $n \geq m_{i}$,

$$
\begin{aligned}
d_{i}\left(\left(T_{n} \circ\right.\right. & \left.\left.T_{n-1} \circ \cdots \circ T_{0}\right)(x), x^{\star}\right) \\
\leq & \alpha_{i} \alpha_{j(i)} \ldots \alpha_{j^{m_{i}(i)}} \alpha(i)^{n-m_{i}} d_{j^{n+1}(i)}\left(x, x^{\star}\right) \\
& +\sum_{k=1}^{m_{i}} \alpha_{i} \alpha_{j(i)} \ldots \alpha_{j^{k-1}(i)} d_{j^{k}(i)}\left(T_{n-k}\left(x^{\star}\right), x^{\star}\right) \\
& +\sum_{k=m_{i}+1}^{n} \alpha_{i} \alpha_{j(i)} \ldots \alpha_{j^{k-1}(i)} d_{j^{k}(i)}\left(T_{n-k}\left(x^{\star}\right), x^{\star}\right)+d_{i}\left(T_{n}\left(x^{\star}\right), x^{\star}\right) \\
\leq & \alpha_{i} \alpha_{j(i)} \ldots \alpha_{j^{m_{i}(i)}} \alpha^{n-m_{i}}(i) q(i, x) \\
& +\sum_{k=1}^{m_{i}} \alpha_{i} \alpha_{j(i)} \ldots \alpha_{j^{k-1}(i)} d_{j^{k}(i)}\left(T_{n-k}\left(x^{\star}\right), x^{\star}\right) \\
& +\sum_{k=m_{i}+1}^{n} \alpha^{k}(i) d_{\beta(i)}\left(T_{n-k}\left(x^{\star}\right), x^{\star}\right)+d_{i}\left(T_{n}\left(x^{\star}\right), x^{\star}\right)
\end{aligned}
$$

Since $\alpha(i)<1$ we have that $\alpha^{n-m_{i}}(i) \rightarrow 0$, as $n \rightarrow \infty$.

The $\operatorname{sum} \sum_{k=1}^{m_{i}} \alpha_{i} \alpha_{j(i)} \ldots \alpha_{j^{k-1}(i)} d_{j^{k}(i)}\left(T_{n-k}\left(x^{\star}\right), x^{\star}\right)$ is finite and we have that $d_{j^{k}(i)}\left(T_{n-k}\left(x^{\star}\right), x^{\star}\right) \rightarrow 0$, as $n \rightarrow \infty$.

For the $\operatorname{sum} \sum_{k=m_{i}+1}^{n} \alpha^{k}(i) d_{\beta(i)}\left(T_{n-k}\left(x^{\star}\right), x^{\star}\right)$ we can apply again Lemma 2.2 from [24], with $a_{k}:=d_{\beta(i)}\left(T_{k}\left(x^{\star}\right), x^{\star}\right)$ and $b_{k}:=\alpha^{k}(i)$. Thus $a_{k} \rightarrow 0$ as $k \rightarrow \infty$ 
and $\sum_{k=0}^{\infty} b_{k}<\infty$. Therefore $\sum_{k=0}^{n} a_{k} b_{n-k} \rightarrow 0$, as $n \rightarrow \infty$. This proves that $d_{i}\left(\left(T_{n} \circ T_{n-1} \circ \cdots \circ T_{0}\right)(x), x^{\star}\right) \rightarrow 0, \quad$ as $n \rightarrow \infty$, for every $i \in I$.

The proof is now complete.

Lemma 2.7. Let $(X, \rightarrow)$ be an L-space and $\left(Y,\left(d_{i}\right)_{i \in I}\right)$ be a sequentially complete Hausdorff gauge space and $j: I \rightarrow I$. Let $x_{n}, x^{\star} \in X$ and $f: X \times Y \rightarrow Y$ be an operator such that

(i) $x_{n} \rightarrow x^{\star}$ as $n \rightarrow \infty$;

(ii) the operator $f(\cdot, y): X \rightarrow X$ is continuous, for all $y \in Y$;

(iii) for every $i \in I$ there exists $\alpha_{i} \in \mathbb{R}_{+}$such that

$$
d_{i}\left(f\left(x, y_{1}\right), f\left(x, y_{2}\right)\right) \leq \alpha_{i} \cdot d_{j(i)}\left(y_{1}, y_{2}\right)
$$

for all $x \in X$ and $y_{1}, y_{2} \in Y$;

(iv) for every $i \in I$ there exists $m_{i} \in \mathbb{N}$ such that for $m \geq m_{i}$ we have

$$
\alpha_{j^{m}(i)} \leq \alpha(i)<1
$$

(v) for every $i \in I$ there exists $p(i)>0$ such that

$$
d_{j^{m}(i)}(y, f(x, y)) \leq p(i)<\infty,
$$

for all $x \in X, y \in Y$ and $m \geq 0$;

(vi) for each $i \in I$ there is $\beta(i) \in I$ such that

$$
d_{j^{m}(i)}\left(y, f\left(x_{n}, y\right)\right) \leq d_{\beta(i)}\left(y, f\left(x_{n}, y\right)\right),
$$

for all $y \in Y, m \geq 0, n \in \mathbb{N}$.

Then the sequence $\left(y_{n}\right)_{n \in \mathbb{N}}$ defined by

$$
y_{0} \in Y, \quad y_{n+1}=f\left(x_{n}, y_{n}\right) \quad(n \in \mathbb{N})
$$

converges to $y^{\star}$, for all $y_{0} \in Y$, where $y^{\star}$ is the unique fixed point of $f\left(x^{\star}, \cdot\right)$.

Proof. We consider

$$
T_{n}, T: Y \rightarrow Y, \quad T_{n}(y)=f\left(x_{n}, y\right), \quad T(y)=f\left(x^{\star}, y\right) .
$$

We have

$$
\begin{aligned}
y_{n+1} & =f\left(x_{n}, y_{n}\right)=T_{n}\left(y_{n}\right)=T_{n}\left(f\left(x_{n-1}, y_{n-1}\right)\right) \\
& =T_{n}\left(T_{n-1}\left(y_{n-1}\right)\right)=\cdots=\left(T_{n} \circ T_{n-1} \circ \cdots \circ T_{0}\right)\left(y_{0}\right) .
\end{aligned}
$$

The proof follows from Lemma 2.6, since

$$
\left(T_{n} \circ T_{n-1} \circ \cdots \circ T_{0}\right)\left(y_{0}\right) \rightarrow T^{\infty}\left(y_{0}\right)=y^{\star}=f\left(x^{\star}, y^{\star}\right),
$$

which means that $y_{n} \rightarrow y^{\star}$. 
Theorem 2.8. Let $(X, \rightarrow)$ be an L-space and $\left(Y,\left(d_{i}\right)_{i \in I}\right)$ be a sequentially complete Hausdorff gauge space and $j: I \rightarrow I$. Let $B: X \rightarrow X$ and $C: X \times Y \rightarrow Y$ be two operators. We suppose that:

(i) $B$ is a PO (we denote by $x^{*}$ its unique fixed point);

(ii) for every $i \in I$ there exists $\alpha_{i} \in \mathbb{R}_{+}$such that

$$
d_{i}\left(C\left(x, y_{1}\right), C\left(x, y_{2}\right)\right) \leq \alpha_{i} \cdot d_{j(i)}\left(y_{1}, y_{2}\right),
$$

for all $x \in X$ and $y_{1}, y_{2} \in Y$;

(iii) for every $i \in I$ there exists $m_{i} \in \mathbb{N}$ such that for $m \geq m_{i}$ we have

$$
\alpha_{j^{m}(i)} \leq \alpha(i)<1
$$

(we denote by $y^{*}$ the unique fixed point of the operator $C\left(x^{*}, \cdot\right)$ );

(iv) for every $i \in I$ there exists $p(i)>0$ such that

$$
d_{j^{m}(i)}(y, C(x, y)) \leq p(i)<\infty,
$$

for all $x \in X, y \in Y$ and $m \geq 0$;

(v) for each $i \in I$ there is $\beta(i) \in I$ such that

$$
d_{j^{m}(i)}\left(y, C\left(B^{n}(x), y\right)\right) \leq d_{\beta(i)}\left(y, C\left(B^{n}(x), y\right)\right),
$$

for all $x \in X, y \in Y, m \geq 0$ and $n \in \mathbb{N}$;

(vi) the operator $C\left(\cdot, y^{\star}\right)$ is continuous in $x^{\star}$.

Then the operator $A: X \times Y \rightarrow X \times Y, A(x, y):=(B(x), C(x, y))$ is a $P O$. Moreover, $F_{A}=\left\{\left(x^{\star}, y^{\star}\right)\right\}$.

Proof. Let $x_{0} \in X$ and $y_{0} \in Y$. We show that

$$
A^{n}\left(x_{0}, y_{0}\right) \rightarrow\left(B^{\infty}\left(x_{0}\right), y^{\star}\right)=\left(x^{\star}, y^{\star}\right),
$$

where $\left\{y^{\star}\right\}=F_{C\left(B^{\infty}\left(x_{0}\right), \cdot\right)}=F_{C\left(x^{\star}, \cdot\right)}$. It is easy to check that

$$
A^{n}\left(x_{0}, y_{0}\right)=\left(B^{n}\left(x_{0}\right), y_{n}\right)
$$

where $x_{n}=B^{n}\left(x_{0}\right), y_{n+1}=C\left(x_{n}, y_{n}\right)$. From (i) we have that $x_{n}=B^{n}\left(x_{0}\right) \rightarrow x^{\star}$. Using again Lemma 2.3 for

$$
T_{n}, \quad T: Y \rightarrow Y, \quad T_{n}(y)=C\left(x_{n}, y\right), \quad T(y)=C\left(x^{\star}, y\right),
$$

we obtain that $y_{n} \rightarrow y^{\star}$ as $n \rightarrow \infty$.

Another extension of Theorem 2.5 relies on the notion of $\varphi$-contraction in gauge spaces (see V. Angelov [3]).

The following result generalize the fiber $\varphi$-contraction theorem given by M. A. Şerban $[30,31]$. We first need a definition and some preparatory results. 
Definition 2.9 (V. Berinde $[7,8]$ ). A function $\varphi: \mathbb{R}_{+} \rightarrow \mathbb{R}_{+}$is called a $(c)$ comparison function if the following conditions hold:

(i) $\varphi$ is monotone increasing;

(ii) there exist two numbers $k_{0}, \alpha, 0<\alpha<1$, and a convergent series of nonnegative terms $\sum_{i=0}^{\infty} v_{k}$ such that

$$
\varphi^{k+1}(t) \leq \alpha \varphi^{k}(t)+v_{k}
$$

for each $t>0$ and each $k \geq k_{0}$.

For some examples of $(c)$-comparison functions see [8].

Lemma 2.10 (V. Berinde $[7,9])$. If $\varphi: \mathbb{R}_{+} \rightarrow \mathbb{R}_{+}$is a (c)-comparison function then:

(a) $\varphi(t)<t$, for each $t>0$;

(b) $\varphi$ is continuous in 0 ;

(c) the series $\sum_{k=0}^{\infty} \varphi^{k}(t)$ converges for each $t \in \mathbb{R}_{+}$;

(d) the sum of the series $s(t)=\sum_{k=0}^{\infty} \varphi^{k}(t)$ is monotone increasing and continuous in 0 ;

(e) $\left(\varphi^{n}(t)\right)_{n \in N}$ converges to 0 , as $n \rightarrow \infty$, for each $t>0$.

Lemma 2.11. Let $\left(X,\left(d_{i}\right)_{i \in I}\right)$ be a sequentially complete Hausdorff gauge space, $j: I \rightarrow I, \varphi_{i}: \mathbb{R}_{+} \rightarrow \mathbb{R}_{+}, i \in I$, are (c)-comparison functions and let $T, T_{n}:$ $X \rightarrow X$ be operators such that:

(i) the sequence $\left(T_{n}\right)_{n \in \mathbb{N}}$ pointwise converges to $T$;

(ii) for each $i \in I$ the function $\varphi_{i}$ is subadditive, i.e. $\varphi_{i}\left(t_{1}+t_{2}\right) \leq \varphi_{i}\left(t_{1}\right)+$ $\varphi_{i}\left(t_{2}\right)$, for all $t_{1}, t_{2} \in \mathbb{R}_{+}$;

(iii) for all $i \in I$ we have

$$
\begin{aligned}
d_{i}\left(T_{n}(x), T_{n}(y)\right) & \leq \varphi_{i}\left(d_{j(i)}(x, y)\right) \\
d_{i}(T(x), T(y)) & \leq \varphi_{i}\left(d_{j(i)}(x, y)\right), \quad \text { for all } x, y \in X ;
\end{aligned}
$$

(iv) for all $i \in I$ there exists a (c)-comparison function $\Phi_{i}$ such that

$$
\sup \left\{\varphi_{j^{n}(i)}(t): n \in \mathbb{N}\right\} \leq \Phi_{i}(t), \quad \text { for each } t \in \mathbb{R}_{+}
$$

(v) for every $i \in I$ there exists $p(i)>0$ such that

$$
d_{j^{m}(i)}\left(x, T_{n}(x)\right) \leq p(i)<\infty \quad \text { and } \quad d_{j^{m}(i)}(x, T(x)) \leq p(i)<\infty,
$$

for all $x \in X, n \in \mathbb{N}$ and $m \geq 0$; 
(vi) for each $i \in I$ there is $\beta(i) \in I$ such that

$$
d_{j^{m}(i)}\left(x, T_{n}(x)\right) \leq d_{\beta(i)}\left(x, T_{n}(x)\right),
$$

for all $x \in X, m \geq 0$ and $n \in \mathbb{N}$.

Then $T, T_{n}, n \in \mathbb{N}$ are POs and the sequence $\left(T_{n} \circ T_{n-1} \circ \cdots \circ T_{0}\right)_{n \in \mathbb{N}}$ pointwise converges to $T^{\infty}$.

Proof. From (ii), (iii) and (iv) we deduce that there exists a unique $x^{\star} \in F_{T}$, $T^{\infty}(x)=x^{\star}$, for all $x \in X$, and $d_{j^{m}(i)}\left(x, x^{*}\right) \leq q(i, x):=q(p(i), x)<+\infty$, for all $m \geq 0$. Let $x \in X$. We have

$$
\begin{aligned}
d_{i}\left(\left(T_{n} \circ\right.\right. & \left.\left.T_{n-1} \circ \cdots \circ T_{0}\right)(x), x^{\star}\right) \\
\leq & d_{i}\left(\left(T_{n} \circ T_{n-1} \circ \cdots \circ T_{0}\right)(x),\left(T_{n} \circ T_{n-1} \circ \cdots \circ T_{0}\right)\left(x^{\star}\right)\right) \\
& +d_{i}\left(\left(T_{n} \circ T_{n-1} \circ \cdots \circ T_{0}\right)\left(x^{\star}\right), T_{n}\left(x^{\star}\right)\right)+d_{i}\left(T_{n}\left(x^{\star}\right), x^{\star}\right) \\
\leq & \cdots \\
\leq & \varphi_{i} \circ \varphi_{j(i)} \circ \cdots \circ \varphi_{j^{n}(i)}\left(d_{j^{n+1}(i)}\left(x, x^{\star}\right)\right) \\
& +\varphi_{i} \circ \varphi_{j(i)} \circ \cdots \circ \varphi_{j^{n-1}(i)}\left(d_{j^{n}(i)}\left(T_{0}\left(x^{\star}\right), x^{\star}\right)\right) \\
& +\cdots+\varphi_{i}\left(d_{j(i)}\left(T_{n-1}\left(x^{\star}\right), x^{\star}\right)\right)+d_{i}\left(T_{n}\left(x^{\star}\right), x^{\star}\right) \\
\leq & \Phi_{i}^{n+1}\left(d_{j^{n+1}(i)}\left(x, x^{\star}\right)\right)+\sum_{k=0}^{n} \Phi_{i}^{k}\left(d_{j^{k}(i)}\left(T_{n-k}\left(x^{\star}\right), x^{\star}\right)\right) \\
\leq & \Phi_{i}^{n+1}\left(s_{i}(p(i, x))\right)+\sum_{k=0}^{n} \Phi_{i}^{k}\left(d_{\beta(i)}\left(T_{n-k}\left(x^{\star}\right), x^{\star}\right)\right),
\end{aligned}
$$

where $s_{i}(t)=\sum_{k=0}^{\infty} \Phi_{i}^{k}(t)$.

Since $\Phi_{i}$ is a $(c)$-comparison function we get $\Phi_{i}^{n+1}\left(s_{i}(p(i, x))\right) \rightarrow 0$, as $n \rightarrow$ $\infty$. For the sum $\sum_{k=0}^{n} \Phi_{i}^{k}\left(d_{\beta(i)}\left(T_{n-k}\left(x^{\star}\right), x^{\star}\right)\right)$ we can apply Lemma 3.1. from M. A. Şerban [30] by taking $a_{k}:=d_{\beta(i)}\left(T_{k}\left(x^{\star}\right), x^{\star}\right)$. Obviously $a_{k} \rightarrow 0$, as $k \rightarrow$ $\infty$ and $\Phi_{i}$ is a $(c)$-comparison function, so $\sum_{k=0}^{n} \Phi_{i}^{k}\left(d_{\beta(i)}\left(T_{n-k}\left(x^{\star}\right), x^{\star}\right)\right) \rightarrow 0$, as $n \rightarrow \infty$. This proves that

$$
d_{i}\left(\left(T_{n} \circ T_{n-1} \circ \cdots \circ T_{0}\right)(x), x^{\star}\right) \rightarrow 0, \quad \text { as } n \rightarrow \infty \text {, for every } i \in I .
$$

The proof is complete.

Lemma 2.12. Let $(X, \rightarrow)$ be an L-space and $\left(Y,\left(d_{i}\right)_{i \in I}\right)$ be a sequentially complete Hausdorff gauge space and $j: I \rightarrow I$. Let $x_{n}, x^{\star} \in X, \varphi_{i}: \mathbb{R}_{+} \rightarrow \mathbb{R}_{+}$, $i \in I$, are (c)-comparison functions and $f: X \times Y \rightarrow Y$ be an operator such that:

(i) $x_{n} \rightarrow x^{\star}$ as $n \rightarrow \infty$;

(ii) for each $i \in I$ the functions $\varphi_{i}$ are subadditive; 
(iii) the operator $f(\cdot, y): X \rightarrow X$ is continuous for all $y \in Y$;

(iv) for every $i \in I$ we have

$$
d_{i}\left(f\left(x, y_{1}\right), f\left(x, y_{2}\right)\right) \leq \varphi_{i}\left(d_{j(i)}\left(y_{1}, y_{2}\right)\right)
$$

for all $x \in X, y_{1}, y_{2} \in Y$;

(v) for every $i \in I$ there exists a (c)-comparison function $\Phi_{i}$ such that

$$
\sup \left\{\varphi_{j^{n}(i)}(t): n \in \mathbb{N}\right\} \leq \Phi_{i}(t), \quad \text { for each } t \in \mathbb{R}_{+} ;
$$

(vi) for every $i \in I$ there exists $p(i)>0$ such that

$$
d_{j^{m}(i)}(y, f(x, y)) \leq p(i)<\infty, \quad \text { where } j: I \rightarrow I,
$$

for all $x \in X, y \in Y$ and for all $m \geq 0$;

(vii) for each $i \in I$ there is $\beta(i) \in I$ such that

$$
d_{j^{m}(i)}\left(y, f\left(x_{n}, y\right)\right) \leq d_{\beta(i)}\left(y, f\left(x_{n}, y\right)\right),
$$

for all $y \in Y, m \geq 0, n \in \mathbb{N}$.

Then the sequence $\left(y_{n}\right)_{n \in \mathbb{N}}$ defined by

$$
y_{0} \in Y, \quad y_{n+1}=f\left(x_{n}, y_{n}\right) \quad(n \in \mathbb{N})
$$

converges to $y^{\star}$, for all $y_{0} \in Y$, where $y^{\star}$ is the unique fixed point of $f\left(x^{\star}, \cdot\right)$.

The proof is essentially the same as of Lemma 2.7 with the modification that instead of Lemma 2.6 we will apply Lemma 2.11.

Theorem 2.13. Let $(X, \rightarrow)$ be an L-space and $\left(Y,\left(d_{i}\right)_{i \in I}\right)$ be a sequentially complete Hausdorff gauge space and $j: I \rightarrow I$. Let $B: X \rightarrow X$ and $C$ : $X \times Y \rightarrow Y$ be two operators and consider $\varphi_{i}: \mathbb{R}_{+} \rightarrow \mathbb{R}_{+}, i \in I,(c)$-comparison functions. We suppose that:

(i) $B$ is a PO (we denote by $x^{*}$ its unique fixed point);

(ii) for every $i \in I$

$$
d_{i}\left(C\left(x, y_{1}\right), C\left(x, y_{2}\right)\right) \leq \varphi_{i}\left(d_{j(i)}\left(y_{1}, y_{2}\right)\right)
$$

for all $x \in X$ and $y_{1}, y_{2} \in Y$;

(iii) for all $i \in I$ there exists a (c)-comparison function $\Phi_{i}$ such that

$$
\sup \left\{\varphi_{j^{n}(i)}(t): n \in \mathbb{N}\right\} \leq \Phi_{i}(t), \quad \text { for each } t \in \mathbb{R}_{+}
$$

(we denote by $y^{*}$ the unique fixed point of the operator $C\left(x^{*}, \cdot\right)$ ); 
(iv) for every $i \in I$ there exists $p(i)>0$ such that:

$$
d_{j^{m}(i)}(y, C(x, y)) \leq p(i)<\infty,
$$

for all $x \in X, y \in Y$ and $m \geq 0$;

(v) For each $i \in I$ there is $\beta(i) \in I$ such that

$$
d_{j^{m}(i)}\left(y, C\left(B^{n}(x), y\right)\right) \leq d_{\beta(i)}\left(y, C\left(B^{n}(x), y\right)\right),
$$

for all $x \in X, y \in Y, m \geq 0$ and $n \in \mathbb{N}$;

(vi) the operator $C$ is continuous.

Then the operator $A: X \times Y \rightarrow X \times Y$ defined by $A(x, y):=(B(x), C(x, y))$ is a PO. Moreover, $F_{A}=\left\{\left(x^{\star}, y^{\star}\right)\right\}$.

Proof. The proof is the same as of Theorem 2.8 with the modification that instead of Lemma 2.6 we will apply Lemma 2.11.

\section{Applications to some functional-integral equations}

Let $(\mathbb{B},+, \mathbb{R} .|\cdot|)$ be a real Banach space, $K \in C\left(\mathbb{R}^{2} \times \mathbb{B}^{2}, B\right), g \in C(\mathbb{R}, \mathbb{B})$ and $h \in C(\mathbb{R}, \mathbb{R})$. In what follow we consider the functional-integral equation $($ see $[5,32])$

$$
x(t)=\int_{-t}^{t} K(t, s, x(s), x(h(s))) d s+g(t), \quad t \in \mathbb{R} .
$$

We have:

Theorem 3.1. We suppose that:

(i) $K \in C\left(\mathbb{R}^{2} \times \mathbb{B}^{2}, \mathbb{B}\right), g \in C(\mathbb{R}, \mathbb{B})$;

(ii) $h \in C(\mathbb{R}, \mathbb{R})$ and $|h(t)| \leq|t|$, for all $t \in \mathbb{R}$;

(iii) there exists $L_{K}>0$ such that

$$
\left|K\left(t, s, u_{1}, v_{1}\right)-K\left(t, s, u_{2}, v_{2}\right)\right| \leq L_{K}\left(\left|u_{1}-u_{2}\right|+\left|v_{1}-v_{2}\right|\right),
$$

for all $t, s \in \mathbb{R}, u_{i}, v_{i} \in \mathbb{B}, i \in\{1,2\}$.

Then:

(a) the equation (1) has in $C(\mathbb{R}, \mathbb{B})$ a unique solution $x^{\star}$;

(b) for all $x_{0} \in C(\mathbb{R}, \mathbb{B})$, the sequence

$$
x_{n+1}(t)=\int_{-t}^{t} K\left(t, s, x_{n}(s), x_{n}(h(s))\right) d s+g(t), \quad t \in \mathbb{R},
$$

uniformly converges to $x^{\star}$ on each compact subset of $\mathbb{R}$. 
Proof. We consider the gauge space $X:=\left(C(\mathbb{R}, \mathbb{B}),\left(d_{n}\right)_{n \in \mathbb{N}}\right)$, where

$$
d_{n}(x, y)=\max _{-n \leq t \leq n}\left(|x(t)-y(t)| \cdot e^{-\tau|t|}\right), \quad \tau>0,
$$

and the operator $B: X \rightarrow X$ defined by

$$
B(x)(t):=\int_{-t}^{t} K(t, s, x(s), x(h(s))) d s+g(t) .
$$

From condition (iii), for each $t \in[-n ; n]$, we have

$$
\begin{aligned}
|B(x)(t)-B(y)(t)| & \leq L_{K} d_{n}(x, y)\left|\int_{-t}^{t} e^{\tau|s|} d s\right| \\
& \leq L_{K} d_{n}(x, y) \int_{-|t|}^{|t|} e^{\tau|s|} d s \\
& \leq \frac{2 L_{K}}{\tau} d_{n}(x, y) e^{\tau|t|} .
\end{aligned}
$$

So,

$$
d_{n}(B(x), B(y)) \leq \frac{2 L_{K}}{\tau} d_{n}(x, y), \quad \text { for all } x, y \in X, n \in \mathbb{N} .
$$

Choosing $\tau>2 L_{K}$ we can apply the result of Colojoară [12] (see also [22, pp. 26], [29, pp. 27]) and thus we obtain the conclusion.

Remark 3.2. In the conditions of Theorem $3.1, B$ is a $P O$ on the gauge space $\left(C(\mathbb{R}, \mathbb{B}),\left(d_{n}\right)_{n \in \mathbb{N}}\right)$.

Let us consider the following functional-integral equation with parameter:

$$
x(t, \lambda)=\int_{-t}^{t} K(t, s, x(s, \lambda), x(h(s), \lambda), \lambda) d s+g(t, \lambda), \quad t \in \mathbb{R}, \lambda \in J \subset \mathbb{R} .
$$

Theorem 3.3. We suppose that:

(i) $J$ is a compact interval of $\mathbb{R}$ and $K \in C\left(\mathbb{R}^{2} \times \mathbb{B}^{2} \times J, \mathbb{B}\right), g \in C(\mathbb{R} \times J, \mathbb{B})$;

(ii) $h \in C(\mathbb{R}, \mathbb{R})$ and $|h(t)| \leq|t|$, for all $t \in \mathbb{R}$;

(iii) $K(t, s, \cdot, v, \lambda), K(t, s, u, \cdot, \lambda) \in C^{1}(\mathbb{B}, \mathbb{B})$ and there exists $M>0$ such that

$$
\left\|D_{3} K(t, s, \cdot, v, \lambda)\right\| \leq M, \quad\left\|D_{4} K(t, s, u, \cdot, \lambda)\right\| \leq M,
$$

for all $t, s \in \mathbb{R}, u, v \in \mathbb{B}, \lambda \in J$;

(iv) $g(t, \cdot) \in C^{1}(J, \mathbb{B})$, for all $t \in \mathbb{R}$.

Under these conditions we have:

(a) the equation (2) has a unique solution $x^{\star}$ in $C(\mathbb{R} \times J, \mathbb{B})$; 
(b) for all $x_{0} \in C(\mathbb{R} \times J, \mathbb{B})$, the sequence

$$
x_{n+1}(t, \lambda)=\int_{-t}^{t} K\left(t, s, x_{n}(s, \lambda), x_{n}(h(s), \lambda), \lambda\right) d s+g(t, \lambda)
$$

$(t \in \mathbb{R}, \lambda \in J)$ converges uniformly, on each compact subset of $\mathbb{R} \times J$, to $x^{\star}$;

(c) $x^{\star}(t, \cdot) \in C^{1}(J, \mathbb{B})$, for all $t \in \mathbb{R}$.

Proof. Let $X=C(\mathbb{R} \times J, \mathbb{B})$ and $B: X \rightarrow X$,

$$
B(x)(t, \lambda):=\int_{-t}^{t} K(t, s, x(s, \lambda), x(h(s), \lambda), \lambda) d s+g(t, \lambda), t \in \mathbb{R}, \lambda \in J
$$

Conclusions (a) and (b) follow as in the proof of Theorem 3.1.

To show (c), we shall use the following heuristic argument. We suppose that there exists $\frac{\partial x^{\star}}{\partial \lambda}$. Then from (2) we have

$$
\begin{aligned}
\frac{\partial x^{\star}}{\partial \lambda}(t, \lambda)= & \int_{-t}^{t}\left(D_{3} K\left(t, s, x^{\star}(s, \lambda), x^{\star}(h(s), \lambda), \lambda\right)\right) \frac{\partial x^{\star}}{\partial \lambda}(s, \lambda) d s \\
& +\int_{-t}^{t}\left(D_{4} K\left(t, s, x^{\star}(s, \lambda), x^{\star}(h(s), \lambda), \lambda\right)\right) \frac{\partial x^{\star}}{\partial \lambda}(h(s), \lambda) d s \\
& \int_{-t}^{t} \frac{\partial K}{\partial \lambda}\left(t, s, x^{\star}(s, \lambda), x^{\star}(h(s), \lambda), \lambda\right) d s+\frac{\partial g}{\partial \lambda}(t, \lambda) .
\end{aligned}
$$

This relation suggests to consider the operator

$$
C: X \times X \rightarrow X, \quad \text { such that }(x, y) \mapsto C(x, y),
$$

where

$$
\begin{aligned}
C(x, y)(t, \lambda):= & \int_{-t}^{t}\left(D_{3} K(t, s, x(s, \lambda), x(h(s), \lambda), \lambda)\right)(y(s, \lambda)) d s \\
& +\int_{-t}^{t}\left(D_{4} K(t, s, x(s, \lambda), x(h(s), \lambda), \lambda)\right)(y(h(s), \lambda)) d s \\
& +\int_{-t}^{t} \frac{\partial K}{\partial \lambda}(t, s, x(s, \lambda), x(h(s), \lambda), \lambda) d s+\frac{\partial g}{\partial \lambda}(t, \lambda)
\end{aligned}
$$

with $t \in \mathbb{R}, \lambda \in J$. In this way we have the triangular operator $A: X \times X \rightarrow X \times X, \quad$ defined by $A(x, y):=(B(x), C(x, y))$. 
From (iii) it follows that $C(x, \cdot): X \rightarrow X, x \in X$, are contractions. Indeed, we have

$$
\begin{aligned}
\mid C & (x, y)(t, \lambda)-C(x, z)(t, \lambda) \mid \\
\leq & \int_{-|t|}^{|t|}\left|\left(D_{3} K(t, s, x(s, \lambda), x(h(s), \lambda), \lambda)\right)(y(s, \lambda)-z(s, \lambda))\right| d s \\
& +\int_{-|t|}^{|t|}\left|\left(D_{4} K(t, s, x(s, \lambda), x(h(s), \lambda), \lambda)\right)(y(h(s), \lambda)-z(h(s), \lambda))\right| d s \\
\leq & M \int_{-|t|}^{|t|}|y(s, \lambda)-z(s, \lambda)| d s+M \int_{-|t|}^{|t|}|y(h(s), \lambda)-z(h(s), \lambda)| d s \\
\leq & 2 M d_{n}(y, z) \int_{-|t|}^{|t|} e^{\tau|s|} d s \\
\leq & \frac{4 M}{\tau} d_{n}(y, z) e^{\tau|t|}, \quad t \in[-n ; n] .
\end{aligned}
$$

Therefore

$$
d_{n}(C(x, y), C(x, z)) \leq \frac{4 M}{\tau} d_{n}(y, z),
$$

for all $x, y, z \in X$. In this case, we can choose $\tau>4 M$ and so the operator $B$ and the operators $C(x, \cdot)$ are contractions. Using Theorem 2.5 we conclude that the operator $A$ is $\mathrm{PO}$ and the sequences

$$
\begin{aligned}
x_{n+1}(t, \lambda)= & \int_{-t}^{t} K\left(t, s, x_{n}(s, \lambda), x_{n}(h(s), \lambda), \lambda\right) d s+g(t, \lambda) \\
y_{n+1}(t, \lambda)= & \int_{-t}^{t}\left(D_{3} K\left(t, s, x_{n}(s, \lambda), x_{n}(h(s), \lambda), \lambda\right)\right)\left(y_{n}(s, \lambda) d s\right. \\
& +\int_{-t}^{t}\left(D_{4} K\left(t, s, x_{n}(s, \lambda), x_{n}(h(s), \lambda), \lambda\right)\right)\left(y_{n}(h(s), \lambda)\right) d s \\
& +\int_{-t}^{t} \frac{\partial K}{\partial \lambda}\left(t, s, x_{n}(s, \lambda), x_{n}(h(s), \lambda), \lambda\right) d s+\frac{\partial g}{\partial \lambda}(t, \lambda)
\end{aligned}
$$

$(t \in \mathbb{R}, \lambda \in J)$ converge uniformly on each compact of $\mathbb{R} \times J$ to $\left(x^{\star}, y^{\star}\right) \in F_{A}$, for all $x_{0}, y_{0} \in X$. But for fixed $x_{0}, y_{0} \in X$ such that $y_{0}=\frac{\partial x_{0}}{\partial \lambda}$ we have that $y_{1}=\frac{\partial x_{1}}{\partial \lambda}$ and by induction we can prove that $y_{n}=\frac{\partial x_{n}}{\partial \lambda}$. So $\frac{\partial x_{n}}{\partial \lambda}$ converges uniformly on each compact of $\mathbb{R} \times J$ to $y^{\star}$. These imply that there exists $\frac{\partial x^{\star}}{\partial \lambda}$ and $\frac{\partial x^{\star}}{\partial \lambda}=y^{\star}$.

Acknowledgement. The authors are grateful to the anonymous referees for the professional comments that improved the final version of this paper. Also, the authors were supported by the the National University Research Council of the Ministry of Education and Research of Romania, Grant CNCSIS 187. 


\section{References}

[1] Andras, Sz., Picard operators and convex contractions. Fixed Point Theory 4 (2003), $121-129$.

[2] Andras, Sz., Fiber $\varphi$-contractions on generalized metric spaces and applications. Mathematica 45 (2003), $3-8$.

[3] Angelov, V., Fixed point theorem in uniform spaces and applications. Czechoslovak Math. J. 37 (112)(1987), 19 - 33.

[4] Angrisani, M. and Clavelli, M., Synthetic approaches to problem of fixed points in metric spaces. Ann. Mat. Pura Appl. (4) 170 (1996), 1 - 12.

[5] Avramescu, C., Sur l'existence des solutions des équations intégrales dans certains espaces fonctionnels (in French). Ann. Univ. Sci. Budapest. Eötvös Sect. Math. 13 (1970), 19 - 34.

[6] Bacotiu, C., Fiber Picard operators on generalized metric spaces. Semin. Fixed Point Theory Cluj-Napoca 1 (2000), 5 - 8.

[7] Berinde, V., Error estimates for a class of $(\delta, \varphi)$-contractions. Babes-Bolyai Univ., Fac. Math. Comput. Sci., Res. Semin., Preprint no. 3 (1994), 3 - 9.

[8] Berinde, V., Error estimates in the approximation of the fixed points for the class of $\varphi$-contractions. Studia Univ. Babes-Bolyai Math. 35 (1990), 86 - 89.

[9] Berinde, V., Contracţii Generalizate şi Aplicaţii (in Romanian). Colecţia Universitaria 2. Baia Mare: Cub Press 22, 1997.

[10] Blumenthal, L. M., Theory and Applications of Distance Geometry. Oxford: Oxford Univ. Press 1953.

[11] Branciari, A., A fixed point theorem of Banach-Caccippoli type on a class of generalized metric spaces. Publ. Math. Debrecen 57 (2000), 31 - 37.

[12] Colojoară, I., Sur un théorème de point fixe dans les espaces uniformes complets (in Romanian). Com. Acad. R. P. Romană 11 (1961), $281-283$.

[13] Dezsö, G., Continuous dependence and derivability with respect to the paprameters of the fixed points. Bull. Appl. Comput. Math., Techn. Univ. Budapest 84 (1998), $245-254$.

[14] Dugundji, J., Topology. Boston: Allyn and Bacon 1966.

[15] Dugundji, J. and Granas, A., Fixed Point Theory. Berlin: Springer 2003.

[16] Fréchet, M., Les Espaces Abstraits. Paris: Gauthier-Villars 1928.

[17] Hadžić, O. and Stanković, B., Some theorems on the fixed point in locally convex spaces. Publ. Inst. Math. (Beograd) 10 (24) (1970), 9 - 19.

[18] Hale, J. K. and Ladeira, L. A. C., Differentiability with respect to delays. J. Diff. Eqs. 92 (1991), 14 - 26.

[19] Hirsch, M. W. and Pugh, C. C., Stable manifolds and hyperbolic sets. Global Analysis (Proc. Sympos. Pure Math. XIV Berkeley, Calif., 1968) 14 (1970), $133-163$. 
[20] Hirsch, M. W., Pugh, C. C. and Shub, M., Invariant Manifolds. Lect. Notes Math. 583. Berlin: Springer 1977.

[21] Jung, C. F. K., On generalized complete metric spaces. Bull. Amer. Math. Soc. 75 (1969), $113-116$.

[22] Kirk, W. A. and Sims, B. (eds.), Handbook of Metric Fixed Point Theory. Dordrecht: Kluwer Acad. Publ. 2001.

[23] de Oliveira, I. C., Variedades Invariantes de Puntos Fixos Hiperbolocos (in Spanish). São Paulo Univ. Preprint 1971.

[24] Rus, I. A., Fiber Picard operators theorem and applications. Studia Univ. Babeş-Bolyai Math. 44 (1999), 89 - 98.

[25] Rus, I. A., A fiber generalized contraction theorem and applications. Mathematica 41 (1999), 85 - 90.

[26] Rus, I. A., Picard operators and applications. Sci. Math. Japon. 58 (2003), 191 -219 .

[27] Rus, I. A., Generalized Contractions and Applications. Cluj-Napoca: Cluj Univ. Press 2001.

[28] Rus, I. A., Sequences of operators and fixed points. Fixed Point Theory 5 (2004), $349-368$.

[29] Rus, I. A., Petruşel, A. and Petruşel, G., Fixed Point Theory 1950-2000: Romanian Contributions. Cluj-Napoca: House of the Book of Science 2002.

[30] Şerban, M. A., Fiber $\varphi$-contraction. Studia Univ. Babeş-Bolyai Math. 44 (1999), $99-108$.

[31] Şerban, M. A., Teoria Punctului Fix Pentru Operatori Definiţi pe Produs Cartezian (in Romanian). Cluj-Napoca: Presa Univ. Clujeană 2002.

[32] Szep, G., On an integral equation with deviating argument. Semin. Fixed Point Theory Cluj-Napoca 1 (2000), 103 - 108.

Received February 13, 2006; revised June 5, 2006 